\title{
Project Social Network: Gestão de Projetos e Equipes, União do PMBOK ao Scrum
}

\author{
Elisrenan S. Barbosa ${ }^{1}$ ，José J. S. F. Luiz² ， Layse S. Santos², Mariano M. \\ Florencio $^{2}$ \\ ${ }^{1}$ Departamento de Computação, Universidade Federal de Sergipe \\ (UFS) -- São Cristóvão, Brasil \\ ${ }^{2}$ Universidade Tiradentes (UNIT) -- Aracaju, Brazil \\ \{elisrenan,jlfjunior8, santoslay3, marianofmendonca\}@gmail.com
}

Abstract. This article presents a tool for decision making based on the union of the Project Management Guide (PMBOK) and the Scrum methodology, called Project Social Network (PSN). Focusing on adapting them to the reality of small and medium-sized enterprises (SMEs). We propose through PSN, a way to manage and develop software, following a simple model and adaptable to the reality of SMEs.

Resumo. Este artigo, apresenta uma ferramenta para tomada de decisão baseado na união do Guia de Gerenciamento de Projetos (PMBOK) e a metodologia Scrum, chamada Project Social Network (PSN). Focando em adaptá-las para a realidade das pequenas e médias empresas (PMES). Propomos através do PSN, uma forma de gerenciar e desenvolver softwares, seguindo um modelo simples e adaptável a realidade das PMEs.

\section{Introdução}

Com a necessidade de garantir a comunicação entre os stakeholders e a entrega de softwares dentro do prazo estimado, foi utilizado como base o Guia de Gerenciamento de Projetos (PMBOK), que segundo (Cruz 2013) pode gerenciar um projeto do início ao fim, em conjunto com a metodologia Scrum que foi projetado para gerenciar equipes de forma ágil e requisitos de projetos que mudam rapidamente (Lei et al. 2017).

\section{Trabalhos Relacionados}

O trabalho de Sadaf et al. 2017, foi o desenvolvimento de um modelo de processo adaptativo (APM), baseado na metodologia ágil XP. Constatou-se depois de um experimento em um ambiente real que é possível desenvolver um produto de forma rápida e eficaz.

Segundo (Alhazmi et al. 2018), as atribuições das tarefas de cada Sprint aos desenvolvedores garantem que cada membro da equipe contribua para seu potencial máximo, e o planejamento do projeto seja otimizado pelo menor tempo possível.

De acordo com (Turner et al. 2010), foca as pequenas empresas precisam de menos documentação e mais desenvolvimento do produto, já as médias de um gerenciamento mais robusto, eficaz e simples. 


\section{Descrição do Project Social Network (PSN)}

O Project Social Network (PSN) é responsável por unir o PMBOK e o Scrum, formando um sistema de gerenciamento de projetos e equipes para tomada de decisões. Ele foi projeto em três camadas: planejamento, gerenciamento e acompanhamento.

$\mathrm{Na}$ camada de planejamento, o gerente de projetos (GP), determina quais processos do PMBOK ele irá utilizar, documenta e libera para a sua equipe Scrum. No gerenciamento a equipe escolhe quais artefatos da metodologia eles têm conhecimento, pois mesmo ela sendo simples de ser entendida, é difícil de ser dominada (Schwaber and Sutherland 2011).

A última camada, é onde estará todas atividades a serem realizadas e seu respectivo andamento, tudo dentro do quadro Scrum. Nessa parte, tanto o GP como o cliente possuem acesso e podem acompanhar a evolução do produto. Por fim, vale ressaltar que o PSN, possui um algoritmo de análise de produtividade, que é capaz de definir quais funcionários estão aptos a determinada tarefa ou requisito e em que média de tempo, ele irá gastar para finalizá-las. Dessa forma, é possível estimar o projeto, como também caso haja algum imprevisto do tipo: troca de funcionário, ou o cliente adicione um novo requisito o algoritmo especifica a pessoa capaz de dar continuidade.

\section{Contribuições}

As MPEs serão benificiadas do PSN, pois nela é possível ter uma documentação simples e adaptada a realidade da empresa. Para analisar o impacto desta ferramenta, retificando ou refutando o que foi desenvolvido, um estudo piloto em uma empresa local está em execução para que se possa constatar, quais lacunas ainda existem e como elas podem ser resolvidas.

\section{References}

Alhazmi, A. and Huang, S. (2018). A decision support system for sprint planning inscrum practice. InSoutheastCon 2018, pages 1-9.

Cruz, F. (2013).Scrum e PMBOK unidos no Gerenciamento de Projetos. Brasport

Lei, H., Ganjeizadeh, F., Jayachandran, P. K., and Ozcan, P. (2017). A statistical analysis of the effects of scrum and kanban on software development projects.Roboticsand Computer-Integrated Manufacturing, 43:59 - 67. Special Issue: Extended PapersSelected from FAIM 2014.

Sadaf, S., Iqbal, S., Saba, A., and KamarMohsin, M. (2017). An extended adaptive process model for agile software development methodology. In2017 International Conference on Intelligent Computing, Instrumentation and Control Technologies (ICICICT),pages 1373-1378.

Schwaber, K. and Sutherland, J. (2011). The scrum guide.Scrum Alliance, 21.

Turner, R., Ledwith, A., and Kelly, J. (2010). Project management in small to mediumsized enterprises: Matching processes to the nature of the firm. International Journalof Project Management, 28(8):744 - 755. IRNOP 2009 in Berlin. 